\title{
On the Energy Gaps Induced by a Semiconducting Substrate in the Graphene Density of States
}

\author{
Sergey Yurevich Davydov ${ }^{1,2} \&$ Alexander Alexandrovich Lebedev ${ }^{1,2}$ \\ ${ }^{1}$ ITMO University, Russia, Saint Petersburg, Kronverksky, Russia \\ ${ }^{2}$ Ioffe Physicotechnical Institute, Russian Academy of Sciences, St. Petersburg, Russia \\ Correspondence: Sergey Yurevich Davidov, ITMO University, Russia, Saint Petersburg, 197101 Kronverksky, \\ Russia. E-mail: sergey_davydov@mail.ru
}

Received: November 5, 2015

Accepted: November 9, 2015

Online Published: October 31, 2015

doi:10.5539/mas.v9n12p234

URL: http://dx.doi.org/10.5539/mas.v9n12p234

\begin{abstract}
The analytical expressions for the densities of states for graphene formed on semiconducting substrates are obtained. The problem on the induced gap is studied thoroughly. It is shown, that graphene electronic spectrum according to the relation between the system's parameters can contain two gaps or one gap, overlapping with the energy gap of substrate. The gaps width dependences on the coupling regimes (tight and weak) are obtained. Numerical estimations are fulfilled for the epitaxial graphene on $6 \mathrm{H}-\mathrm{SiC}\{0001\}$.
\end{abstract}

Keywords: graphene, epitaxy, silicon carbide, graphene-substrate bonding

\section{Introduction}

The unique properties of graphene are well-known and, of a large extent, theoretically based now (see, e. g., (Castro Neto A.H., et al., 2008; Kotov V.N., et al., 2012; Falkovsky I.V. and Vassilevich D.V., 2012; Cooper D.R., et al., 2011; Wu Y.H., et al., 2010; Haas J., et al., 2008; Seyller Th., et al., 2008) and references therein). Graphene coupled with substrate and, as a rule, formed on it is called epitaxial graphene (Castro Neto A.H., et al., 2008; Cooper D.R., et al., 2011; Haas J., et al., 2008; Seyller Th., et al., 2008). The silicon carbide substrates $6 \mathrm{H}\{0001\}$ and $4 \mathrm{H}\{0001\}$ are used most often, since thermodesorption of silicon atoms from these surfaces leads to the formation of the graphene film (Haas J., et al., 2008; Seyller Th., et al., 2008). The results of such a process are critically depended from what face ( $\mathrm{Si}$ or $\mathrm{C}$ ) silicon atoms desorption has taken place (Mathieu C., et al., 2011; Srivastava N., et al., 2012; Goler S., et al., 2013; Jayasekera T., et al., 2011; Deretzis I. and La Magna A., 2011). If the $\mathrm{SiC}(0001)$, or Si-face, substrate has been used, than the obtained graphene monolayer is separated from the substrate by the so called buffer leyer, which is covalently bonded to substrate.

For the $\mathrm{SiC}(000 \overline{1})$, or $\mathrm{C}$-face substrate situation is not quite clear. In the most part of the studies it is demonstrated that the graphene monolayer only weakly bound with substrate is formed.

However, there are opposite data (see, e. g., (Mathieu C., et al., 2011; Srivastava N., et al., 2012) and references therein) that the interaction of the first carbon atoms layer with substrate is tight. Note also, that one of the epitaxial graphene type is the so called quasi-free standing graphene, which realizes due to intercalation of some stranger atoms (say, atomic hydrogen) just under the outer carbon layer to destroy the covalent bonding between graphene and substrate. Thus, the nature of the graphene-substrate interaction is of the great significance since it is determined the one-sheet graphene characteristics. One of the most significant feature for the one-layer epitaxial graphene is the presence or absence of the energy gaps in it density of states (DOS). Actually, gap of the appropriate width, or, better, tuned width is the pledge of the graphene usage in the device structures. Here we put forward rather simple model which permits us to obtain graphene DOS in an analytical form and solve the problem on the gap existence. To obtain the epitaxial graphene DOS we employ the simple artificial method firstly used in (Davydov S.Yu., 1978), where one takes as the bare Green function those for the single adatom instead of free atom.

\section{Method}

In what follows we will use the Green's functions method together with the model Hamiltonian approach to the problem. In our opinion this scheme is the most adequate for the study of epitaxial graphene since it permits to clarify the substrate effect on the graphene DOS in a simple and straightforward way. 
Let us consider isolated atom with the energy level $\varepsilon_{a}$, occupied site $i$ of the adsorbed layer. The corresponding Hamiltonian is:

$$
H_{0}=\sum_{k} \varepsilon_{k} c_{k}^{+} c_{k}+\varepsilon_{a} a_{i}^{+} a_{i}+\sum_{k}\left(V_{k a} c_{k}^{+} a_{i}+V_{a k} a_{i}^{+} c_{k}\right)
$$

Here $\varepsilon_{k}$ is the dispersion low for the substrate electrons, $V_{k a}$ is the matrix element of the adatom's state $\left|a_{i}\right\rangle$ interaction with the substrate state $|\mathbf{k}\rangle, c_{k}^{+}\left(c_{k}\right)$ is the creation (annihilation) operator for the electron in the state $|\mathbf{k}\rangle, a_{i}^{+}\left(a_{i}\right)$ the same operators but for the adatom's state $\left|a_{i}\right\rangle$. The Green function $g_{i j}=\left\langle a_{j}|g| a_{i}\right\rangle$, corresponding to the Hamiltonian (1) has the form (see e. g., Davydov S.Yu., 2011; Davydov S.Yu. and Troshin S.V., 2007; Anderson P.W., 1961):

$$
g_{i j}=\delta_{i j}\left(\omega-\varepsilon_{a}-\Lambda(\omega)+i \Gamma(\omega)\right)^{-1} .
$$

Here $\delta_{i j}$ is the Kronecker symbol, the quasilevel half-width function is

$$
\Gamma(\omega)=\pi V^{2} \rho_{s}(\omega),
$$

where $\rho_{s}(\omega)$ is the substrate DOS, and the quasilevel shift function is

$$
\Lambda(\omega)=\frac{1}{\pi} P \int_{-\infty}^{\infty} \frac{\Gamma\left(\omega^{\prime}\right) d \omega^{\prime}}{\omega-\omega^{\prime}} d \omega^{\prime} .
$$

Note that we introduced here the mean value of the matrix element putting $V=\sqrt{\left\langle\left|V_{k a}\right|^{2}\right\rangle}$, where $\langle\ldots\rangle$ stands for the k-average of $V_{k a}$ (Haldane F.D.M. and Anderson P.W., 1976).

Introduce now the operator for the electron interadatom transitions $T_{i j}=\left\langle a_{j}|T| a_{i}\right\rangle$ with amplitude $t$, which acts between the adjacent adatoms, occupied nearest neighbor sites $i$ and $j$. To find corresponding Green function $G_{i j}$ we use Dyson equation of the form:

$$
G_{i j}=g_{i j}+g_{i l} T_{l m} G_{m j}
$$

Take the "zero" adatom at the site $(0,0)$ and three its nearest neighbors at the sites: $1-a(-\sqrt{3} / 2,1 / 2), 2-$ $a(\sqrt{3} / 2,1 / 2), 3-a(0,-1)$, where $a=1.42 \AA$ is the nearest neighbor separation. Then we get

$$
G_{00}=g_{00}+g_{00} t\left(G_{10}+G_{20}+G_{30}\right) .
$$

Taking into account the transforming properties of the Green functions $G_{0 i}$, we obtain

$$
\begin{aligned}
& G_{00}^{-1}(\omega, \mathbf{k})=\Omega-\Lambda(\omega)+i \Gamma(\omega) \mp t f(\mathbf{k}), \\
& f^{2}(\mathbf{k})=3+2 \cos \left(k_{x} a \sqrt{3}\right)+4 \cos \left(k_{x} a \sqrt{3} / 2\right) \cos \left(3 k_{y} a / 2\right) .
\end{aligned}
$$

Here $\mathbf{k}$ is a wave vector for graphene, $\Omega=\omega-\varepsilon_{a}$, upper sign corresponds to the $\pi^{*}$-band $(\bar{\Omega} \equiv \Omega-\Lambda(\omega)>0)$, lower sign corresponds to the $\pi$-band $(\bar{\Omega}<0)$. In what follows we will accept the low-energy approximation of the dispersion of the form (Castro Neto A.H., et al., 2008; Davydov S.Yu., 2011):

$$
\varepsilon_{ \pm}(q)=\varepsilon_{a} \pm \frac{3}{2} t a|\mathbf{q}|
$$

where $\mathbf{q}=\mathbf{K}-\mathbf{k}, \mathbf{K}=a^{-1}(2 \pi / 3 \sqrt{3}, 2 \pi / 3)$ is the Dirac point vector. Eq.(8) is correct only for small $q$. Finally we get

$$
G_{00}^{-1}(\omega, q)=\bar{\Omega}+i \Gamma(\omega) \mp \frac{3 t}{2}(a q) .
$$

Further we will omit the site indexes and introduce index "g" which demonstrates that this value corresponds graphene.

Now the DOS corresponding Green function (9) is 


$$
\rho_{g}(\omega, q)=\frac{1}{\pi} \frac{\Gamma(\omega)}{[\bar{\Omega} \mp(3 t / 2)(a q)]^{2}+\Gamma^{2}(\omega)} .
$$

By integrating $\rho_{g}(\omega, q)$ over $q$ vectors from the first Brillouin zone and introducing the cutoff vector $q_{B}$, we find

$$
\rho_{g}(\omega)=\frac{1}{\pi \xi^{2}}\left[\Gamma(\omega) \ln \frac{(\bar{\Omega} \mp \xi)^{2}+\Gamma^{2}(\omega)}{\bar{\Omega}^{2}+\Gamma^{2}(\omega)}+2 \bar{\Omega}\left(\tan ^{-1} \frac{\bar{\Omega}}{\Gamma(\omega)}-\tan ^{-1} \frac{\bar{\Omega} \mp \xi}{\Gamma(\omega)}\right)\right]
$$

where $\xi \equiv 3 \operatorname{taq}_{B} / 2$.

\section{Results and Analysis}

\subsection{Induced Energy Gap}

To describe semiconducting substrate we take Haldane-Anderson model (Haldane F.D.M. and Anderson P.W., 1976; Davydov S.Yu. and Troshin S.V., 2007), where for the substrate DOS $\rho_{s}$ the following expression is accepted:

$$
\begin{aligned}
\rho_{s}(\omega) & =\rho_{s}, \quad|\omega| \geq E_{g} / 2, \\
& =0, \quad|\omega|<E_{g} / 2,
\end{aligned}
$$

where $E_{g}$ is the substrate energy gap (energy zero coincides with the center of the gap), $\rho_{s}=$ const. Using (3) and (4), we arrive at

$$
\begin{aligned}
& \Gamma(\omega)=\Gamma, \quad|\omega| \geq E_{g} / 2, \\
&=0, \quad|\omega|<E_{g} / 2, \\
& \Lambda(\omega)=\frac{\Gamma}{\pi} \ln \left|\frac{\omega-E_{g} / 2}{\omega+E_{g} / 2}\right| .
\end{aligned}
$$

Now let us consider substrate energy gap domain taking $\Gamma(\omega) \rightarrow 0$. Note that $\Lambda(\omega)$ does not become zero in this energy interval. Going to the dimensionless quantities

$$
B(x)=\varepsilon_{g}(x-\eta)-\lambda(x),|x|<1,
$$

where $x=2 \omega / E_{g}, \eta=2 \varepsilon_{a} / E_{g}, \varepsilon_{g}=E_{g} / 2 \Gamma$, and $\lambda(x)=\pi^{-1} \ln (1-x) /(1+x)$, we find from Eq.(11) the condition for the gap opened in the epitaxial graphene DOS:

$$
\tan ^{-1} \frac{B(x)}{s}-\tan ^{-1} \frac{B(x) \mp v}{s}=0, s=0^{+},
$$

where $v=\xi / \Gamma$. Since the arc tans in Eq.(16) can be equal only to $( \pm \pi / 2)$, it is necessary and sufficient to fulfill the following inequality:

$$
B(x)(B(x) \mp v)>0,
$$

where minus sign stands for $B(x)>0$, plus sign stands for $B(x)<0$. Inequality (17) is satisfied for two cases: (i) $B(x)>v$, (ii) $B(x)<-v$. Both these conditions can be transformed to one, i. e.

$$
|B(x)|>v .
$$




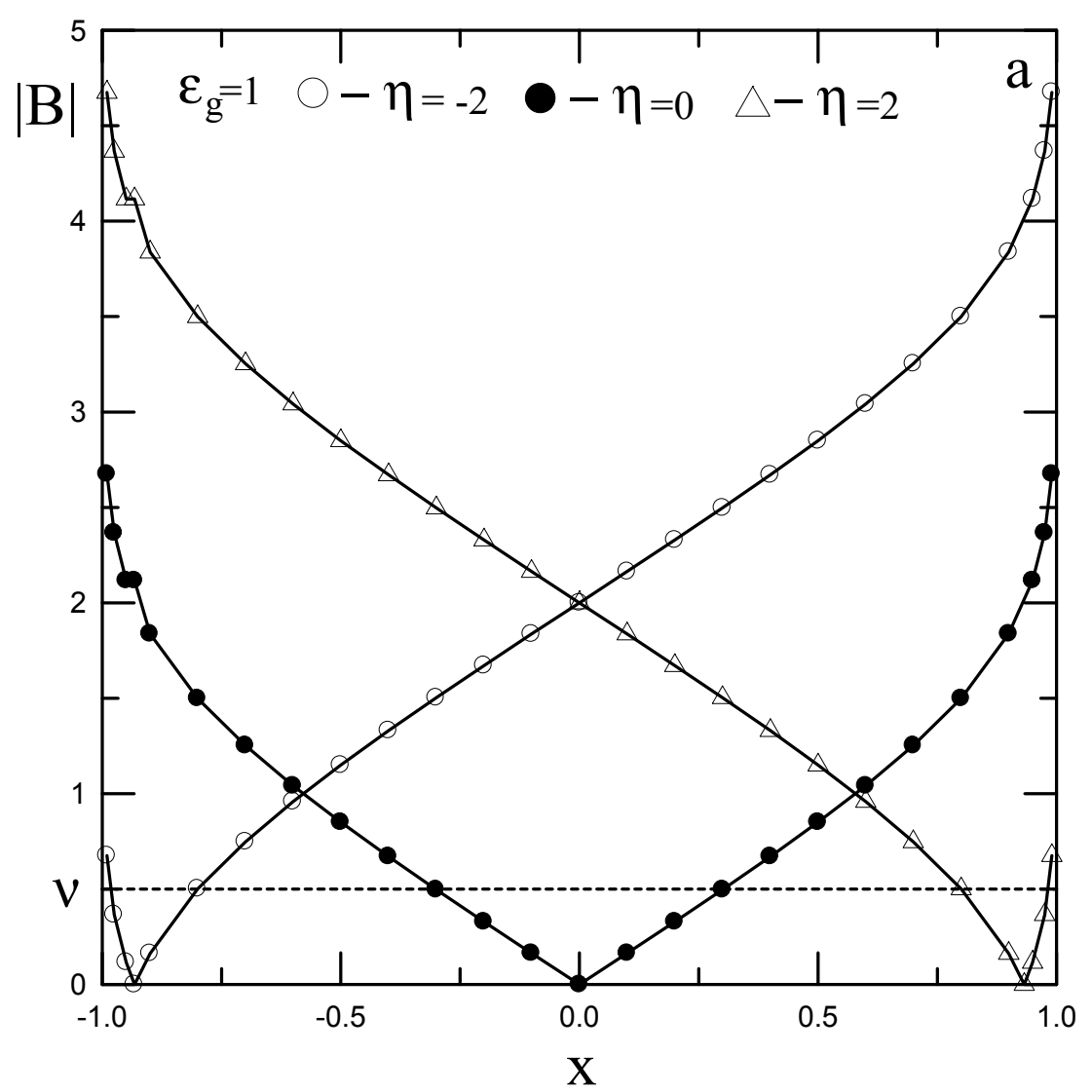

Figure 1a. $|B|$ v.s. $|x|$ for $\varepsilon_{g}=1$ and $\eta=-2,0,2$.

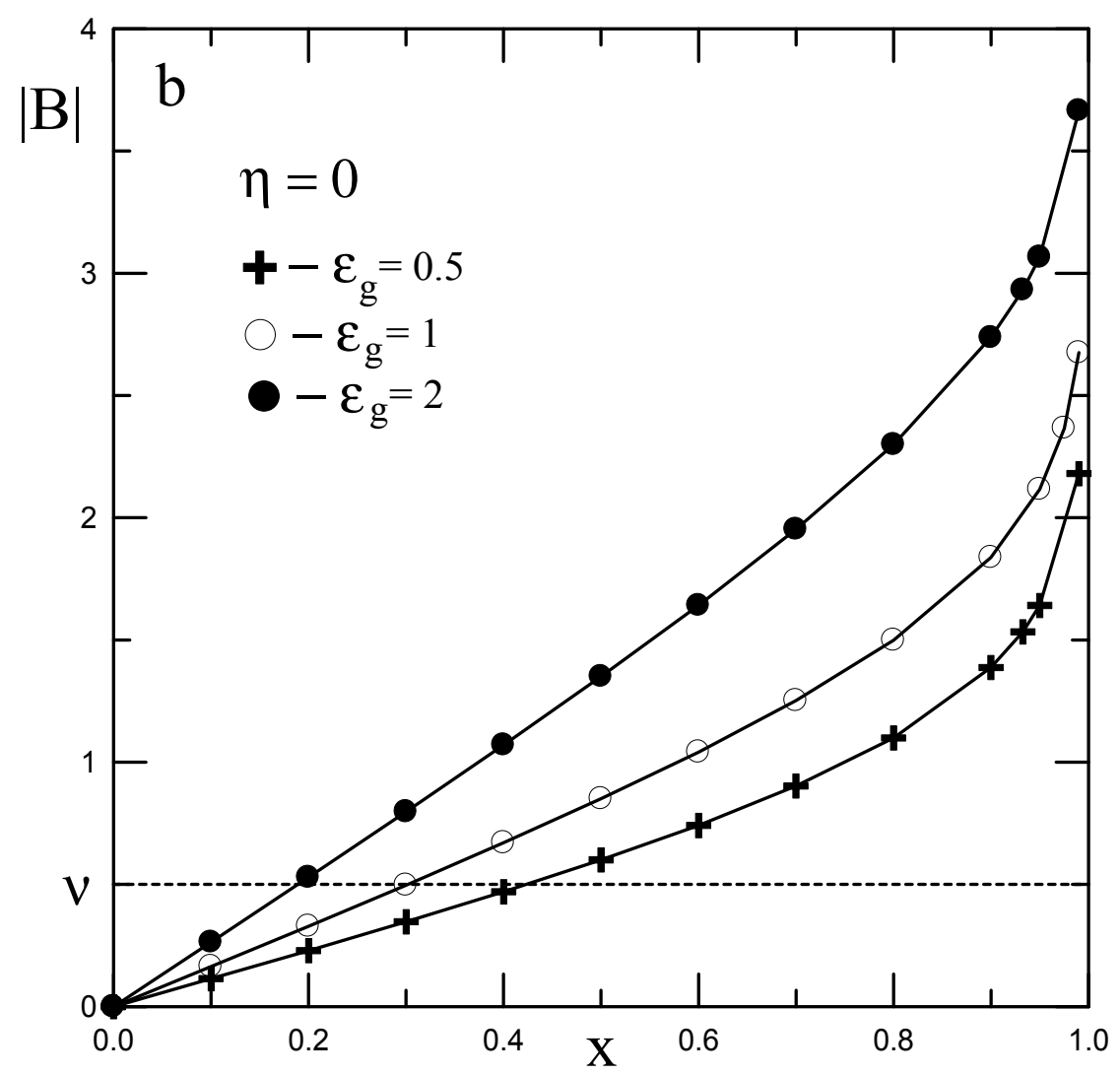

Figure 1b. $|B|$ v.s. $|x|$ for $\eta=0$ and $\varepsilon_{g}=0.5,1,2$. 
Figure 1 demonstrates graphical method of the gap width determination. For the tight graphene-substrate bonding when the graphene atoms interaction with the substrate atoms is much stronger than the interaction between the graphene atoms, i. e. for $v<<1$. We see from Figure 1a that at the limit $v \rightarrow 0$ the graphene gap $\Delta \rightarrow E_{g}$. The nature of such a result is quite clear, since at this limit the epitaxial graphene DOS is proportional to the single adatom DOS $\rho_{g 0}(\omega)$, corresponding to the Green function (2) and equals to $\pi^{-1} \Gamma(\omega) /\left[\bar{\Omega}+\Gamma^{2}(\omega)\right]$, which goes to zero for the substrate gap energy region. More exactly, for $v<<1$ there are two equal symmetrical gaps divided by the narrow band of the midgap states around the Dirac point, since this latter is determined by the equation $B(x)=0$. It is worthy to note here that this equation gives the local states within the substrate energy gap for the single carbon adatom (see Eq.(2) and Haldane F.D.M. and Anderson P.W., 1976; Davydov S.Yu. and Troshin S.V., 2007).

In the opposite case for the weak graphene-substrate bonding $(v>>1)$ two gaps in the vicinities of the valence and conduction bands appear. Here must be taken into account that the asymptotes $|B(x)| \rightarrow \infty$ which are arisen from $\lambda(x) \rightarrow \pm \infty$ for $x \rightarrow \mp 1$ have their origin in the substrate DOS approximation (12). If the more realistic model for the DOS (without steps) would be accepted then these discrepancies have to vanish. Thus, for the $v \gg>1$ case the narrow gaps near the band edges can be theoretical artefacts. Thus, at the limit $v \rightarrow \infty$ the epitaxial graphene DOS transforms to the free graphene $\operatorname{DOS} \rho_{g}^{\text {free }}(\omega)=2|\omega| / \xi^{2}$ for $|\omega|<\xi$ and 0 otherwise (Davydov S.Yu., 2012).

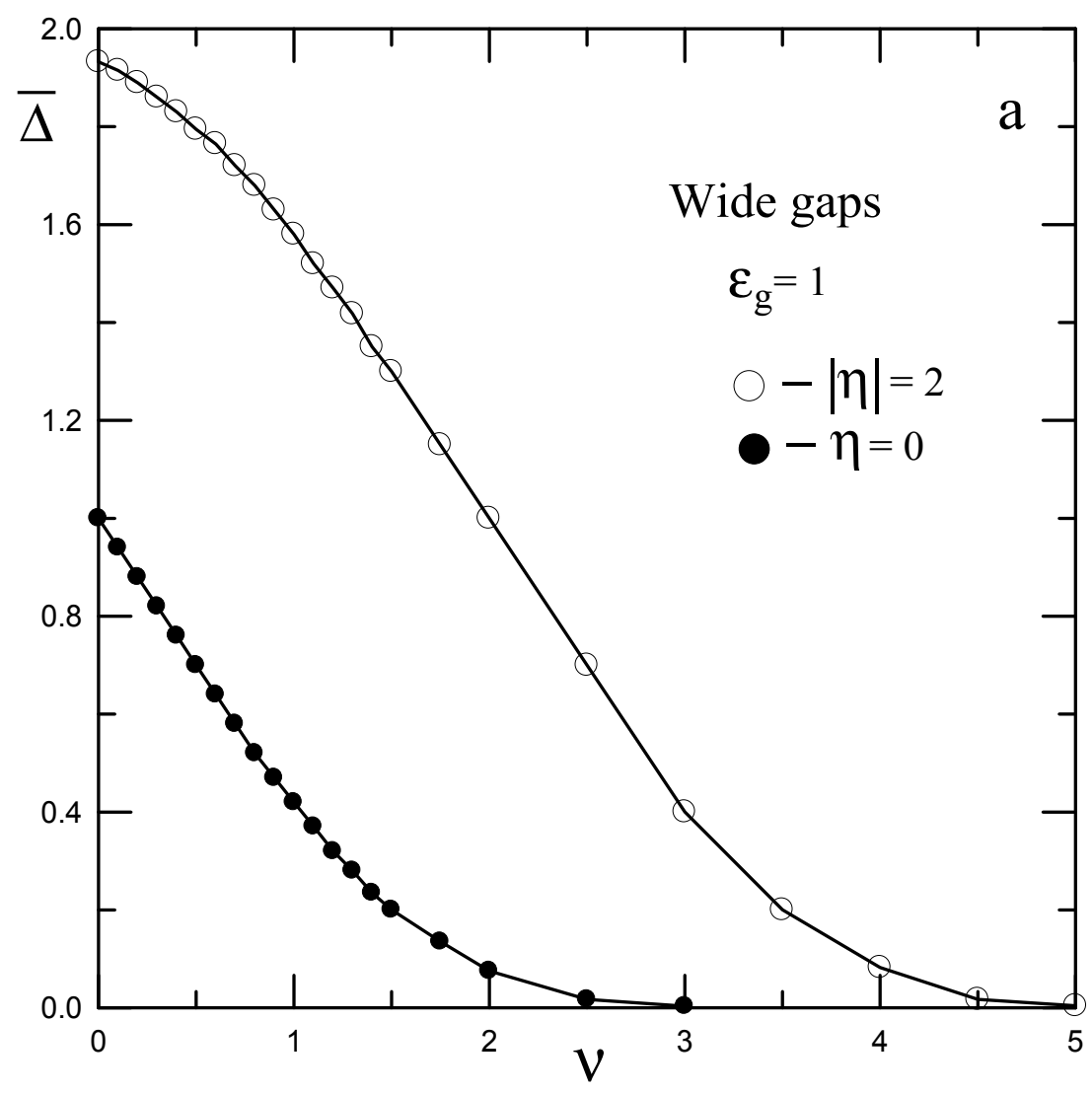

Figure 2a. Wide gaps: $\bar{\Delta}$ v.s. $v$ for $\varepsilon_{g}=1$ and $\eta=0, \mp 2$. 


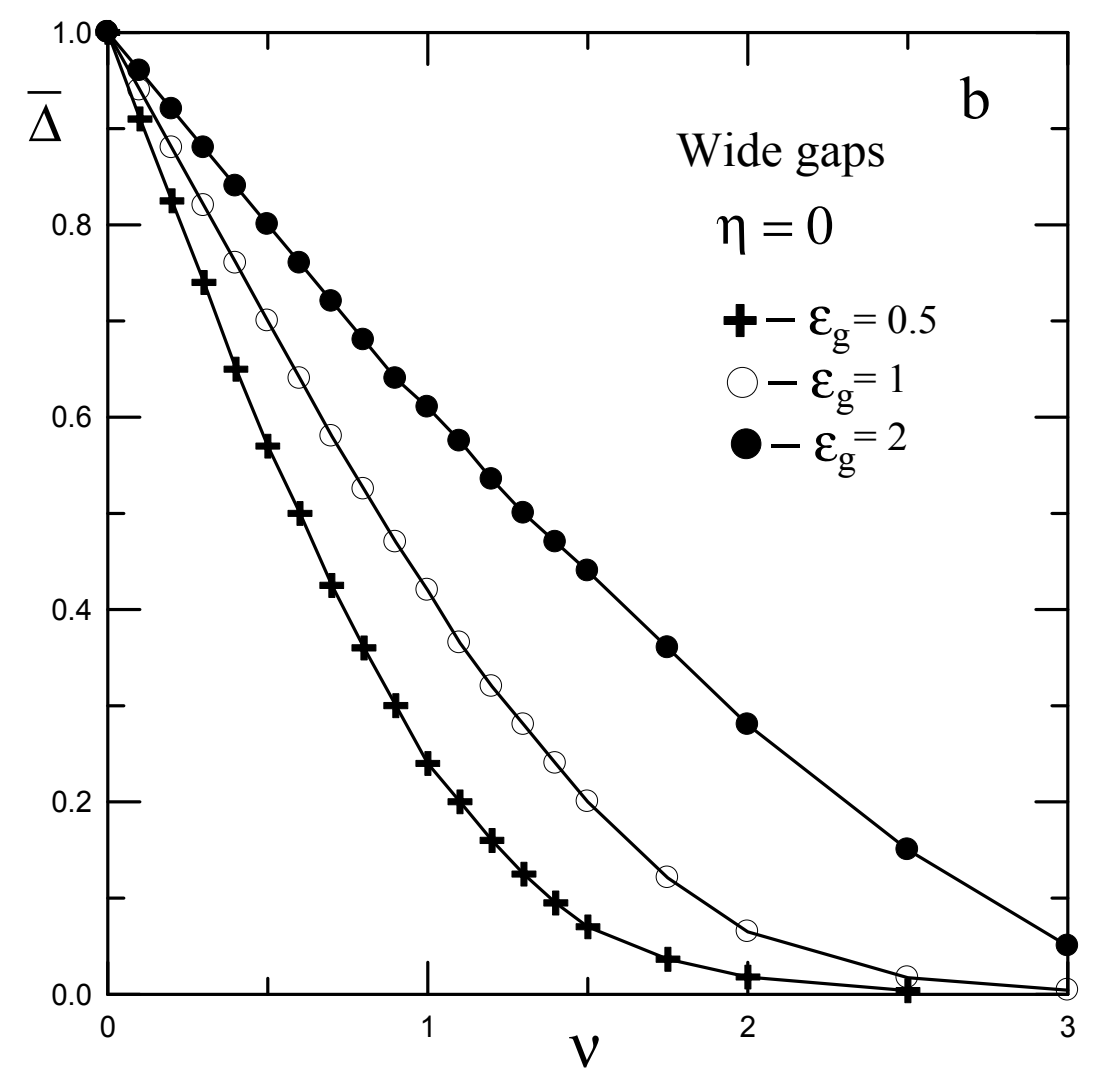

Figure 2b. Wide gaps: $\bar{\Delta}$ v.s. $v$ for $\eta=0$ and $\varepsilon_{g}=0.5,1,2$.

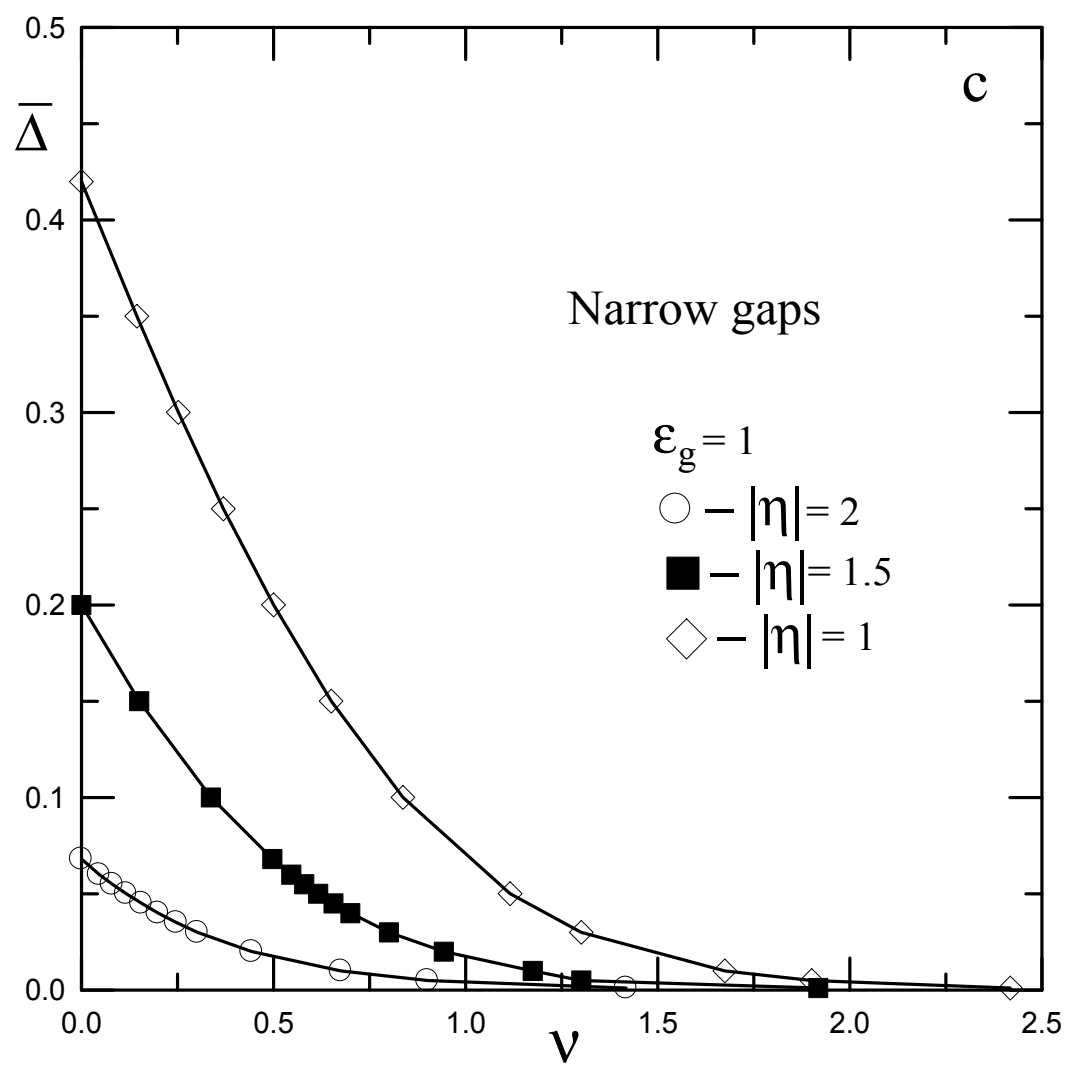

Figure 2c. Narrow gaps: $\bar{\Delta}$ v.s. $v$ for $\varepsilon_{g}=1$ and $|\eta|=2,1.5,1$. 
Figure 2 demonstrates the calculated results for the reduced graphene energy gap $\bar{\Delta}=2 \Delta / E_{g}$ dependences on the $v$ value for the number of the problem parameters. From Figure 2a for the wide gaps it is clear that with $v$ increasing from the zero value two gaps are realized (see also Figure 1a). For $\eta=0$ they are symmetric and their widths decrease. In the case $|\eta|>1$ and rather small $v$ we have both narrow and wide gaps. For $\eta<0$ the narrow gap is localized near the valence band edge, while the wide gap coincides with the conduction band. For $\eta>0$ one has opposite situation. It is easy to show that for the case $|\eta| \gg>1, \eta<0$ the Dirac point is equal to $x_{-} \approx-1+2 \exp \left(-\pi \varepsilon_{g}|\eta|\right)$. Thus, the maximal gap width is $2 \exp \left(-\pi \varepsilon_{g}|\eta|\right)$, or $E_{g} \exp \left(-\pi\left|\varepsilon_{a}\right| / \Gamma\right)$. For the case $|\eta| \gg>1, \eta>0$ the Dirac point takes the form $x_{+} \approx 1-2 \exp \left(-\pi \varepsilon_{g} \eta\right)$, which gives $2 \exp \left(-\pi \varepsilon_{g} \eta\right)$ for the maximal gap width. Note also that for $|\eta|<<1$ we find that the Dirac point is $x_{l 0} \approx \eta /\left[1+\left(2 / \pi \varepsilon_{g}\right)\right]$.

Figure $2 \mathrm{~b}$ presents the $\bar{\Delta}$ increase with the $\varepsilon_{g}=E_{g} / 2 \Gamma$ value (see also Figure $1 \mathrm{~b}$ ). That is not surprising since the $|B(x)|$ increases with $\varepsilon_{g}$. Figure 2c indicates the narrow gaps shrinkage with the $v$ increasing, $i . e$., with the transition from the tight graphene-substrate bonding regime to the weak one.

\section{Discussion}

\subsection{Numerical Estimations}

As far as the authors know the data on the epitaxial graphene energy gaps available (Seyller Th., et al., 2008; Goler S., et al., 2013; Jayasekera T., et al., 2011; Zhou S.Y., et al., 2007; Mattausch A. and Pankratov O., 2007; Vitali L., et al., 2008; Pankratov O., et al., 2010; Kim S., et al., 2008; Huang B., et al., 2011) are very contradictory and display $\Delta$ from zero to $E_{g}$. The same is concerned to other characteristics, say, the Dirac point positions. For example, for the graphene on the C-face of SiC papers (Zhou S.Y., et al., 2007; Mattausch A. and Pankratov O., 2007) give the Dirac point position at $0.4 \mathrm{eV}$ below the Fermi level, while in (de Heer W.A., et al., 2010) this value is equal to $0.3 \mathrm{eV}$ and in (Emtsev K.V., et al., 2008; Berger C., et al., 2006) to $0.2 \mathrm{eV}$. Moreover, authors of (Lin Y.M., et al., 2010; Miller D.L., et al., 2009; Hofmann T., et al., 2011) find that the Dirac point lies above the Fermi level. Nonetheless, we try to make some numerical estimations here for the $6 \mathrm{H}-\mathrm{SiC}\{0001\}$ substrate.

Taking $\chi=3.45 \mathrm{eV}$ (Davydov S.Yu., 2007) and $E_{g}=3 \mathrm{eV}$, we obtain that the substrate energy gap center corresponds to the energy $\chi+E_{g} / 2=-4.95 \mathrm{eV}$ below vacuum level. Let the Dirac point for the $6 \mathrm{H}-\mathrm{SiC}(0001)$ substrate lies at $0.4 \mathrm{eV}$ below the Fermi level (Zhou S.Y., et al., 2007; Mattausch A. and Pankratov O., 2007). Since the corresponding work function $\phi_{g 1}=3.75 \mathrm{eV}$ (Mattausch A. and Pankratov O., 2007), we get the Dirac point at $0.8 \mathrm{eV}$ above the substrate gap center. For the $6 \mathrm{H}-\mathrm{SiC}(000 \overline{1})$ substrate the Fermi level coincides with the Dirac point and the corresponding work function $\phi_{g 2}=5.33 \mathrm{eV}$ (Mattausch A. and Pankratov O., 2007). Then the Dirac point has the energy $-0.38 \mathrm{eV}$ below the substrate gap center.

Now we have to determine the epitaxial graphene gaps from the equation $B(x)=0$. To fulfill it we have to estimate the problem parameters. Taking into account the results of (Davydov S.Yu., 2011), we may equate matrix element $V$ to Harrison's covalent energy for the $\sigma$-bond of the two $\mathrm{sp}^{3}$ orbitals $V_{2}=3.22\left(\hbar^{2} / \mathrm{md}^{2}\right)$ (Harrison W.A., 1983), where $\hbar$ is the reduced Planck constant, $m$ is the free electron mass, $d$ is the graphene-substrate bond length, equal to $2 \AA$ (Mattausch A. and Pankratov O., 2007). Then we obtain $V \sim 6 \mathrm{eV}$. Since only one third carbon atom of the buffer layer participates in covalent bonding with the substrate $\mathrm{Si}$ atoms, we take the effective interaction $V_{\text {eff }} \sim V / 3 \sim 2 \mathrm{eV}$. Estimating $\rho_{s}$ to be $\sim 0.25 \mathrm{eV}^{-1}$ (valence band of the width $\sim 15 \mathrm{eV}$ (Mercer J.L., 1996; Persson C. and Lindefelt U., 1997) contains 4 electrons), we get $\Gamma \sim 3 \mathrm{eV}$. Then $\varepsilon_{g} \sim 0.5$. Since $t \sim 3 \mathrm{eV}$ (Castro Neto A.H., et al., 2008), we have $\xi \sim 3 \mathrm{eV}$, where we put $\left(3 a q_{B} / 2\right) \sim 1$. Consequently, $v=\xi / \Gamma \sim 1$. Using equation $B(x)=0$ for Si-face we find $\eta \approx 1.71$. Finally, we have

the lower wide gap at $(-1.50,-0.20) \mathrm{eV}$ and the upper narrow gap at $(1.46,1.50) \mathrm{eV}$. This latter one is most likely the artefact. For the $6 \mathrm{H}-\mathrm{SiC}(0001)$ we find $\eta \approx-0.58$, provided the carbon sheet is the buffer layer. Then we find the lower narrow gap to be at $(-1.50,-1.31) \mathrm{eV}$, while the upper wide gap at $(0.87,1.50) \mathrm{eV}$. Here the lower gap seems to be artifact. As to quasi-free standing graphene, the estimation shows the gapless electronic structure.

Thus, in this study we have considered a row of possible energy schemes wich can be realized for the epitaxial graphene. Underline that the simple model approach used here permits us to obtain the quite reasonable results in an analytical form. Moreover, such an approach can be easely complicated for the account of additional experimental data. Unfortunately, the lack of this data prevents more thorough investigation of the epitaxial graphene problem.

\section{Acknowledgments}

This article was prepared as part of the "1000 laboratories" program with the support of Saint-Petersburg 
National Research University of Information Technologies, Mechanics and Optics (University ITMO) and was supported in part by the Russian Foundation for Basic Research (project 12-02-00165a) and by the Government of Russian Federation (grant 074-U01).

\section{References}

Anderson, P. W. (1961). Localized Magnetic States in Metals. Physical Review, 124(41). http://dx.doi.org/10.1103/PhysRev.124.41

Berger, C., et al. (2006). Electronic Confinement and Coherence in Patterned Epitaxial Graphene. Science, 312(5777), 1191-1196. http://dx.doi.org/10.1126/science.1125925

Castro, Neto A. H., et al. (2008). The electronic properties of graphene. Reviews of Modern Physics, 81(109), 55.

Cooper, D. R., et al. (2011). Experimental review of graphene. ISRN Condensed Matter Physics, 2012(501686), 56. http://dx.doi.org/10.5402/2012/501686

Davydov, S. Yu. (1978). Electron state of adatoms at high coverages. Sov. Phys. Solid State, 20, 1013.

Davydov, S. Yu. (2011). Electronic states in epitaxial graphene fabricated on silicon carbide. Semiconductors, 45(8), 1070-1076. http://dx.doi.org/10.1134/S1063782611080057

Davydov, S. Yu. (2011). On charge transfer in the adsorbed molecules-graphene monolayer-SiC substrate system. Semiconductor, 45(5), 618-622. http://dx.doi.org/10.1134/S1063782611050083

Davydov, S. Yu. (2012). Adsorption-induced energy gap in the density of states of single-sheet graphene. Semiconductors, 46(2), 193-198. http://dx.doi.org/10.1134/S1063782612020066

Davydov, S. Yu., \& Troshin, S. V. (2007). Adsorption on metals and semiconductors: Anderson-Newns and Haldane-Anderson models. Physics of the Solid State, 49(8), 1583-1588. http://dx.doi.org/10.1134/S1063783407080318

Davydov, S. Yu. (2007). On the electron affinity of SiC polytypes. Semiconductors, 41(6), 696-698. http://dx.doi.org/10.1134/S1063782607060152

de Heer W. A., et al. (2010). Epitaxial graphene electronic structure and transport. Journal of Physics D: Applied Physics, 43(37), 374007. http://dx.doi.org/10.1088/0022-3727/43/37/374007

Deretzis I., \& La Magna A. (2011). Single-layer metallicity and interface magnetism of epitaxial graphene on SiC. Applied Physical Letters, 98, 023113. http://dx.doi.org/10.1063/1.3543847

Emtsev K.V., et al. (2008). Interaction, growth, and ordering of epitaxial graphene on $\mathrm{SiC}\{0001\}$ surfaces: A comparative photoelectron spectroscopy study. Physical Review B, 77, 155303. http://dx.doi.org/10.1103/PhysRevB.77.155303

Falkovsky, I. V., \& Vassilevich, D. V. (2012). Quantum Field Theory in Graphene. International Journal of Modern Physics A, 27, 1260007, 12. http://dx.doi.org/10.1142/S0217751X1260007X

Goler S., et al. (2013). Revealing the atomic structure of the buffer layer between $\mathrm{SiC}(0001)$ and epitaxial graphene. Carbon, 51, 249-254. http://dx.doi.org/10.1016/j.carbon.2012.08.050

Haas J., de Heer W. A., \& Conrad, E. H. (2008). The growth and morphology of epitaxial multilayer graphene. Journal of Physics: Condensed Matter, 20, 323202. http://dx.doi.org/10.1088/0953-8984/20/32/323202

Haldane, F. D. M., \& Anderson, P. W. (1976). Simple model of multiple charge states of transition-metal impurities in semiconductors. Physical Review B, 13, 2553. http://dx.doi.org/10.1103/PhysRevB.13.2553

Harrison, W. A. (1983). Theory of two-center bond. Physical Review B, 27, 3592. http://dx.doi.org/10.1103/PhysRevB.27.3592

Hofmann, T., et al. (2011). Hole-channel conductivity in epitaxial graphene determined by terahertz optical-Hall effect and midinfrared ellipsometry. Applied Physical Letters, 98, 041906. http://dx.doi.org/10.1063/1.3548543

Huang, B., Xiang, H. J., \& Wei, H. J. (2011). Controlling doping in graphene through a SiC substrate: A first-principles study. Phys. Rev. B 83, 161405 (R). http://dx.doi.org/10.1103/PhysRevB.83.161405

Jayasekera, T., Xu, S., Kim, K.W., \& Nardelli, M.B. (2011). Electronic properties of the graphene $/ 6 \mathrm{H}-\mathrm{SiC}\left(0001^{-}\right)$ $\begin{array}{llllll}\text { interface: A first-principles study. Physical Review } B, & 84, & 035442 .\end{array}$ http://dx.doi.org/10.1103/PhysRevB.84.035442

Kim, S., Ihm, J., Choi, H. J., \& Son, Y. W. (2008). Origin of Anomalous Electronic Structures of Epitaxial 
Graphene on Silicon Carbide. Physical Review Letters, 100, 176802. http://dx.doi.org/10.1103/PhysRevLett.100.176802

Kotov, V. N., et al. (2012). Electron-Electron Interactions in Graphene: Current Status and Perspectives. Reviews of Modern Physics, 84(1067), 55. http://dx.doi.org/10.1103/RevModPhys.84.1067

Lin, Y. M., et al. (2010). Multicarrier transport in epitaxial multilayer graphene. Applied Physical Letters, 97, 112107. http://dx.doi.org/10.1063/1.3485671

Mathieu, C., et al. (2011). Microscopic correlation between chemical and electronic states in epitaxial graphene on SiC(000-1). Physical Review B, 83, 235436. http://dx.doi.org/10.1103/PhysRevB.83.235436

Mattausch, A., \& Pankratov, O. (2007). Ab Initio Study of Graphene on SiC. Physical Review Letters, 99, 076802. http://dx.doi.org/10.1103/PhysRevLett.99.076802

Mercer, J. L. (1996). Tight-binding models for compounds: Application to SiC. Physical Review B, 54, 4650. http://dx.doi.org/10.1103/PhysRevB.54.4650

Miller, D. L., et al. (2009). Observing the Quantization of Zero Mass Carriers in Graphene. Science, 324(5929), 924-927. http://dx.doi.org/10.1126/science.1171810

Pankratov, O., Hendel, S., \& Bockstedle, M. (2010). Electron spectrum of epitaxial graphene monolayers. Physical Review B, 82, 121416. http://dx.doi.org/10.1103/PhysRevB.82.121416

Persson, C., \& Lindefelt, U. (1997). Relativistic band structure calculation of cubic and hexagonal SiC polytypes. Journal of Applied Physics, 82, 5496. http://dx.doi.org/10.1063/1.365578

Seyller, Th., et al. (2008). Epitaxial graphene: a new material. Physica Status Solidi B, 245(7), 1436-1446. http://dx.doi.org/10.1002/pssb.200844143

Srivastava, N., He, G., Luxmi, \& Feenstra, R. M. (2012). Interface Structure of Graphene on $\operatorname{SiC}(0001)$. Physical Review B,0 85, 041404. http://dx.doi.org/10.1103/PhysRevB.85.041404

Vitali L., et al. (2008). Spatial modulation of the Dirac gap in epitaxial graphene. Surface Science, 602(22), L127-L130. http://dx.doi.org/10.1016/j.susc.2008.09.030

Wu, Y. H., Yu, T., \& Shen, Z. X. (2010). Two-dimensional carbon nanostructures: Fundamental properties, synthesis, characterization, and potential applications. Journal of Applied Physics, 108, 071301. http://dx.doi.org/10.1063/1.3460809

Zhou, S. Y., et al. (2007). Substrate-induced bandgap opening in epitaxial graphene. Nature Materials, 6, 770-775. http://dx.doi.org/10.1038/nmat2003

\section{Copyrights}

Copyright for this article is retained by the author(s), with first publication rights granted to the journal.

This is an open-access article distributed under the terms and conditions of the Creative Commons Attribution license (http://creativecommons.org/licenses/by/3.0/). 